\title{
Structural Biology of Bacterial Transcription
}

Transcription is the major control point of gene expression and RNA polymerase (RNAP), conserved from bacteria to man, is the central enzyme of transcription. Our long term goal is to understand the mechanism of transcription and its regulation. Determining three-dimensional structures of RNAP and its complexes with DNA, RNA, and regulatory factors, is an essential step. We focus on highly characterized prokaryotic RNAPs. The basic elements of the transcription cycle, initiation, elongation, and termination, were elucidated through study of prokaryotes. A detailed structural and functional understanding of the entire transcription cycle is essential to explain the fundamental control of gene expression and to target RNAP with small-molecule antibiotics. At every stage of the transcription cycle, RNAP function is modulated by interactions with extrinsic regulatory factors. Assembling and crystallizing transcription complexes containing extrinsic regulators presents challenges for structural biology. Due to recent advances, cryo-electron microscopy (cryo-EM) now offers a route to structural and mechanistic characterization of these large assemblies. We are using cryo-electron microscopy, in combination with X-ray crystallography and other approaches, to provide a complete characterization of the bacterial transcription cycle.

Seth Darst

James Chen

Jin Young Kang

Elizabeth Campbell

The Rockefeller University 